\title{
Effects of Acute Sacral Neuromodulation at Different Frequencies on Bladder Overactivity in Pigs
}

\author{
Xing $\operatorname{Li}^{1,2,3,4}$, Limin Liao ${ }^{1,2,3,4}$, Guoqing Chen ${ }^{1,2,3,4}$, Zhaoxia Wang $^{1,2,3,4}$, Han Deng ${ }^{1,2,3,4}$ \\ ${ }^{1}$ Department of Urology, Capital Medical University, Beijing, China \\ ${ }^{2}$ Department of Urology, China Rehabilitation Research Centre, Beijing, China \\ ${ }^{3}$ Beijing Key Laboratory of Neural Injury and Rehabilitation, Beijing, China \\ ${ }^{4}$ Center of Neural Injury and Repair, Beijing Institute for Brain Disorders, Beijing, China
}

Purpose: We investigated the effects of different stimulation frequencies on the inhibition of bladder overactivity by sacral neuromodulation (SNM) in pigs.

Methods: Implant-driven stimulators were used to stimulate the S3 spinal nerve in 13 pigs. Cystometry was performed by infusing normal saline (NS) or acetic acid (AA). SNM (pulse width, $210 \mu \mathrm{sec}$ ) at frequencies ranging from 5 to $50 \mathrm{~Hz}$ was conducted at the intensity threshold at which observable perianal and/or tail movement was induced. Multiple cystometrograms were performed to determine the effects of different frequencies on the micturition reflex.

Results: AA-induced bladder overactivity significantly reduced the bladder capacity (BC) to $34.4 \% \pm 4.7 \%$ of the NS control level $(354.4 \pm 35.9 \mathrm{~mL})(\mathrm{P}<0.05)$. During AA infusion, SNM at $5 \mathrm{~Hz}$ did not significantly change the BC $(48.1 \% \pm 6.9 \%$ of the NS control level) $(\mathrm{P}>0.05)$, but $\mathrm{SNM}$ at 15,30 , and $50 \mathrm{~Hz}$ significantly increased the BC to $54.5 \% \pm 7.1 \%, 55.2 \% \pm 6.5 \%$, and $57.2 \% \pm 6.1 \%$ of the NS control level $(\mathrm{P}<0.05)$, respectively. No significant differences were found among the results obtained using frequencies of 15,30 , and $50 \mathrm{~Hz}(\mathrm{P}>0.05)$.

Conclusions: This study demonstrated that $15 \mathrm{~Hz}$ was an appropriate frequency for SNM and that frequencies higher than 15 $\mathrm{Hz}$ did not lead to better surgical outcomes.

Keywords: Frequency; Neuromodulation; Urinary Bladder, Overactive; Sacral Nerve Stimulation

- Fund Support: This study was funded by the National Natural Science Foundation of China (81570688), Beijing Municipal Science \& Technology Commission (Z151100001615055), and Beijing Natural Science Foundation (7153179).

- Research Ethics: All applicable international, national, and/or institutional guidelines for the care and use of animals were followed. All experiments in this study were approved by the animal care and use committee at Capital Medical University (AEEI-2016-113).

- Conflict of Interest: No potential conflict of interest relevant to this article was reported.

\section{- HIGHLIGHTS}

- The study investigated the effects of different stimulation frequencies on the inhibition of bladder overactivity by sacral neuromodulation (SNM) in pigs.

- It demonstrated that $15 \mathrm{~Hz}$ was an appropriate frequency for SNM and that frequencies higher than $15 \mathrm{~Hz}$ did not lead to better surgical outcomes.

\section{INTRODUCTION}

Overactive bladder syndrome is characterized by urgency that is usually accompanied by frequency, with or without urge incontinence. It seriously impacts the patient's quality of life. Many patients do not respond to medications such as anticho-

Corresponding author: Limin Liao (iD https://orcid.org/0000-0002-7092-6576 Department of Urology, China Rehabilitation Research Center, No 10. Jiaomen Beilu, Fengtai District, Beijing 100068, China

E-mail: lmliao@263.net / Tel: +86-10-8756-9043/ Fax:+86-10 -6757-0492

Submitted: November 20, 2016 / Accepted after revision: November 28, 2016
cC) $(7)$ This is an Open Access article distributed under the terms of the Crecommons.org/licenses/by-nc/4.0/) which permits unrestricted non-commercial use, distribution, and reproduction in any medium, provided the original work is properly cited. 
linergic drugs owing to lack of adherence, efficacy, or tolerability, as well as due to the side effects of these drugs [1]. Patients who are refractory to these treatments are likely to respond to sacral neuromodulation (SNM) or sacral nerve stimulation [2]. Appropriate patients can be accurately identified during the pre-implant test stimulation, and the implantation criteria are based on clinical and subjective improvement. An assessment of the stimulation parameters should be performed to achieve better clinical outcomes. Some experts believe that increased stimulation frequency might lead to more efficient bladder inhibition [3]. Currently, the frequency used in clinical practice of SNM is $10-20 \mathrm{~Hz}$ [4-7].

Identification of the optimal stimulation frequency is important in clinical neuromodulation. Many studies have reported the effects of frequency on the inhibition of bladder overactivity in animals. Some studies have shown that tibial nerve stimulation (TNS) and foot stimulation at either a low $(5 \mathrm{~Hz})$ or a high $(30 \mathrm{~Hz})$ frequency were effective in inhibiting bladder overactivity $[8,9]$. Other studies have indicated that the efficacy of pudendal nerve stimulation (PNS) is frequency-dependent [1012]. The goals of this study were to determine whether bladder inhibition or excitation could be induced by SNM using different stimulation frequencies. We investigated the effects of acute SNM delivered by an implant-driven stimulator (SacralStim, General Stim Inc., Hangzhou, China) on the micturition reflex in pigs.

\section{MATERIALS AND METHODS}

\section{Device Introduction}

The SacralStim system implanted in the pigs consisted of 3 subsystems: a stimulator, a stimulating electrode with 6 contact points, and an extension cable (Fig. 1). The implant stimulator (approximately $50 \mathrm{~mm} \times 50 \mathrm{~mm} \times 7 \mathrm{~mm}$ ) allows minimally invasive surgery when implanted in the body. It is encapsulated in a titanium case. Titanium is inert and biocompatible, so it will not oxidize or be toxic to the tissue. A high-density battery is used to power the electronic system. In the body, it is electrically connected to the proximal end of a lead about $80 \mathrm{~mm}$ in length through an extension cable. The distal end of the cable, where 6 inline insulated and independent electrodes are located, targets the S3 nerve. This electronic system generates programmed electrical pulses, which propagate through the leads and reach the nerves for modulation. The implant supports radiofrequency wireless communication with the external controller (an Android device). The communication is 2-way (i.e., the external controller can send data to an implant, and the implant can send data back to the external controller). The stimulation parameters are a voltage of $0-10 \mathrm{~V}$, a frequency rate of $5-110 \mathrm{~Hz}$, and pulse width of 30-630 $\mu$ sec. These parameters can be adjusted quickly using the remote control.

\section{Surgical Implantation}

Experiments were conducted in 13 mature Guizhou miniature pigs ( 7 male, 6 female; weight range, $25-28 \mathrm{~kg}$; age range, 10-13 months). The animal care and use committee at Capital Medical University approved all protocols involving the use of animals in this study (AEEI-2016-113). The pigs were anesthetized by an intramuscular injection of ketamine hydrochloride ( 10 $\mathrm{mg} / \mathrm{kg}$ ) and maintained with isoflurane (2\%-5\% in oxygen) by a trachea cannula connected to an anesthesia machine (Matrix VMR, Midmark, Dayton, OH, USA). The heart rate and the blood oxygen level were monitored using an electrocardioscope monitor (Cardell 9500, Midmark). The pigs were placed in the prone position on a heating pad to maintain a constant body temperature.
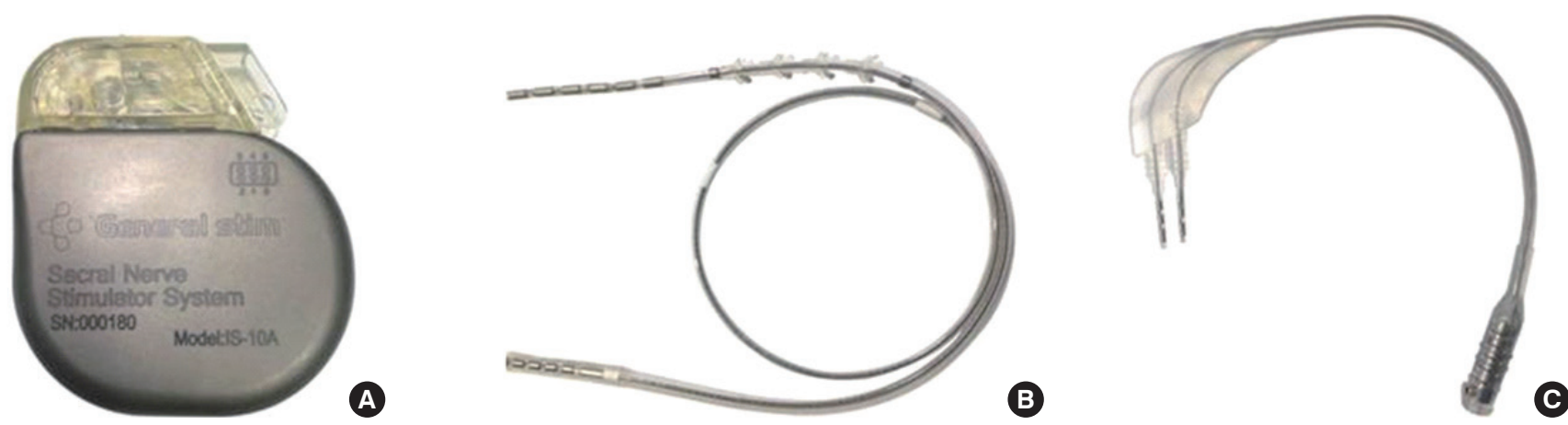

Fig. 1. The pictures of real products: stimulator (A), stimulating electrode with 6 contact points (B), and extension cable (C). 
After shaving the buttock and back followed by disinfection, we made a 5- to 6-cm incision from the middle of the posterior superior iliac spine to the tail. According to our experience in anatomy, only the $\mathrm{S} 3$ foramen of pigs can be penetrated by the localizing needle extending from the SacralStim device. The localizing needle was then replaced with a guide wire, and a dilating trocar was passed following the stimulating electrode, with 6 contact points introduced (Model ISL-280620, SacralStim; Fig. 2). After successful placement, an external neurostimulator (ModelGSPO-10A, SacralStim) was used to verify the appropriate motor responses in the perianal area and/or tail by gradually increasing the voltage. An extension cable (Model EL1006 , SacralStim) was then tunneled to separate subcutaneous pockets in the upper buttock and connected to the stimulator (Model IS-10A SacralStim; Fig. 2). The incisions were then closed using sutures. Radiographs were taken to identify the appropriate position after implantation (Fig. 3). Enrofloxacin $(0.1 \mathrm{mg} / \mathrm{kg})$ was administered intramuscularly to prevent infection for a week, and tilidine hydrochloride $(0.1 \mathrm{mg} / \mathrm{kg})$ was administered intramuscularly to relieve pain for at least 3 days.

\section{Cystometry and SNM Testing}

After implantation for a week, experiments with acute SNM were conducted. The pigs were anaesthetized by an intramuscular injection of ketamine hydrochloride $(10 \mathrm{mg} / \mathrm{kg})$. After approximately 1 hour, anesthesia was induced with intravenous
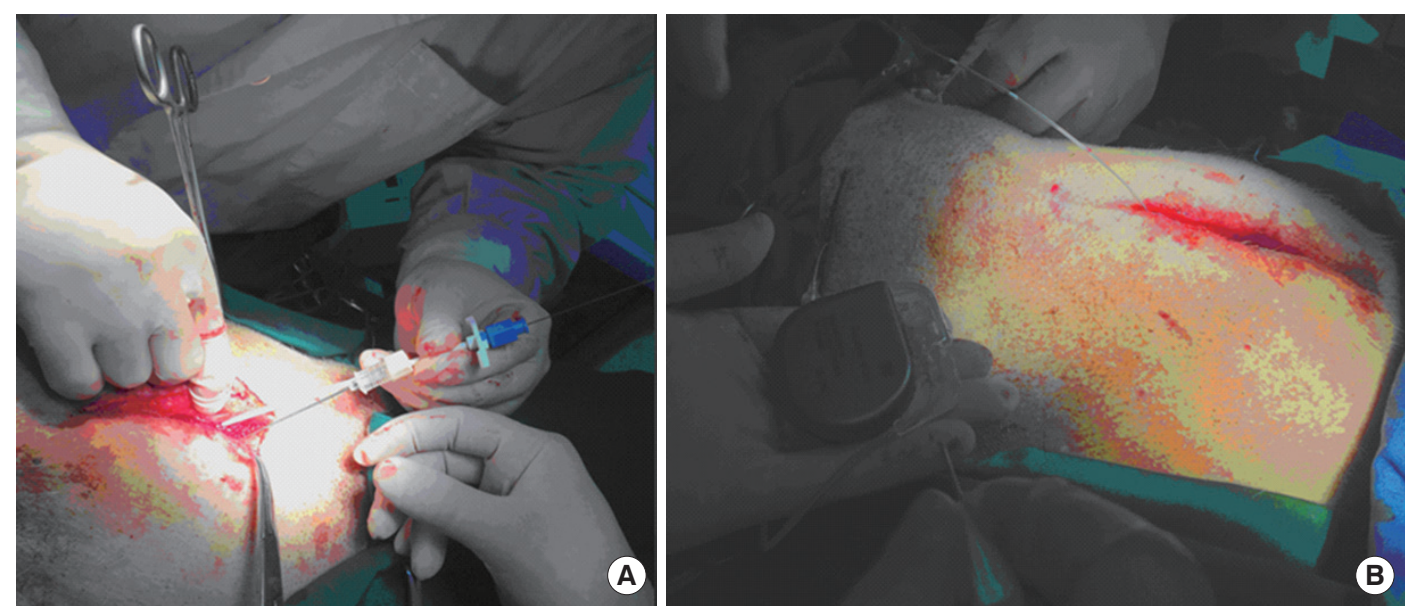

Fig. 2. The implantation process of SacralStim (General Stim Inc., Hangzhou, China): placing the stimulation electrodes (A) and connecting the extension cable with the stimulator (B).
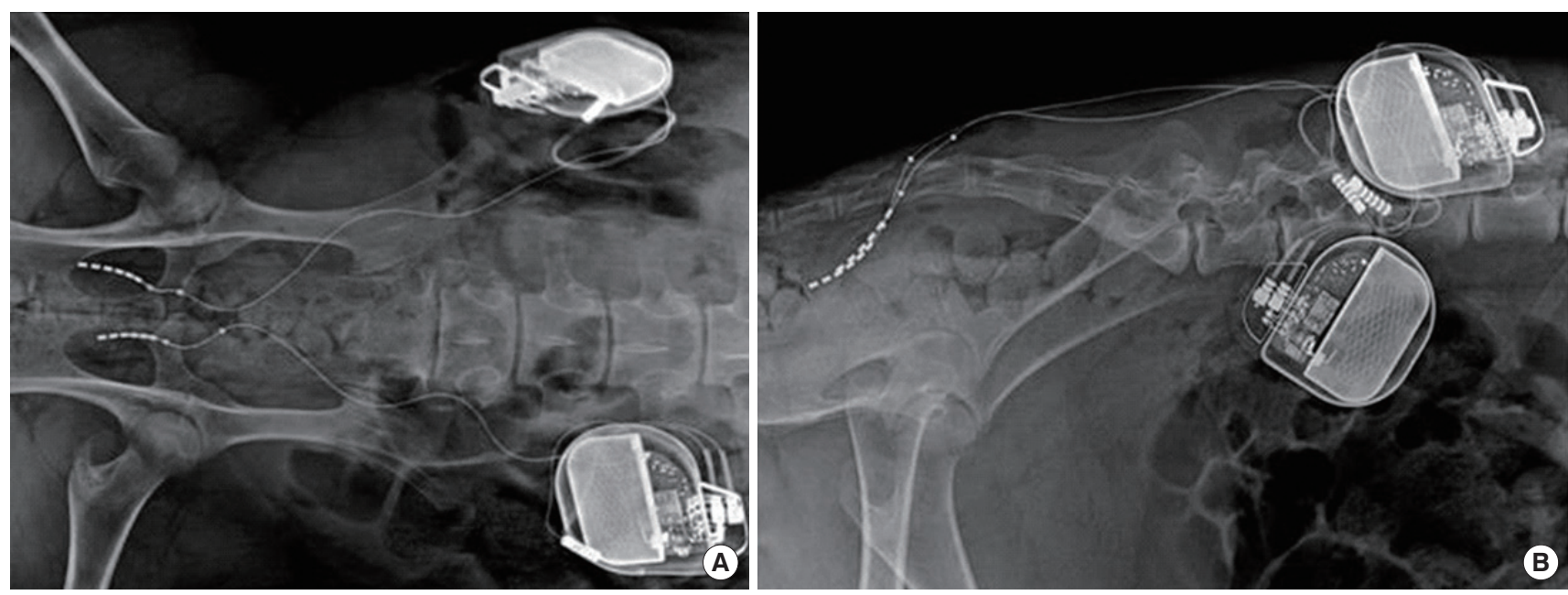

Fig. 3. Radiographs show the leads passed through the S3 foramen: anteroposterior (A) and lateral (B). 
a-chloralose $(62.5 \mathrm{mg} / \mathrm{kg})$ and maintained with intravenous a-chloralose (50 mg/kg/hr) [13]. Respiration was supported by a breathing machine (Matrix 3000, Midmark), and the heart rate and the blood oxygen level were monitored by the electrocardioscope monitor throughout the experiment. Fluid was administered via the ear vein. Body temperature was maintained through the use of a heating pad.

The urethra was exposed via the inferior margin of the pubis. An 8F 2-lumen catheter (Cook Company, Spencer, IN, USA) was inserted into the bladder through the urethra. The catheter was then secured by a ligature around the urethra. One lumen of the catheter was used for infusing the bladder with either normal saline (NS) or acetic acid (AA) at a rate of $30 \mathrm{~mL} / \mathrm{min}$ after connecting to a pump. The other lumen was connected to a pressure transducer (Andromeda Urodynamic System, Taufkirchen/Potzdam, Germany) to measure the bladder pressure. Methods, definitions, and units conformed to the International Continence Society standards [14]. After completing the preparation, the stimulation began.

\section{Stimulation Protocol}

Before stimulation, impedance was measured to check device integrity. Uniphasic rectangular pulses (pulse width, $210 \mu \mathrm{sec}$ ) were delivered to the sacral nerve. The intensity threshold for inducing perianal and/or tail movement was determined by gradually increasing the stimulation intensity. Bladder capacity (BC) was used for testing the inhibitory effect of the stimulator. The bladder was drained before each experiment. After the appearance of the first sharp, large-amplitude bladder contraction, bladder infusion with NS or AA was stopped, and this infusion volume was defined as the BC. After emptying the bladder, we first performed 2 or 3 cystometrograms (CMGs) with NS without stimulation to obtain the control BC. Then, after emptying the bladder, 5\% AA was infused into the bladder to irritate and induce bladder overactivity for 3-5 CMGs. After the BC stabilized, SNM (5- to 50- $\mathrm{Hz}$ frequencies) was applied during sequential CMGs. The actual intensity threshold varied among pigs, from 1 to $6 \mathrm{~V}$. The bladder was emptied after each CMG, and a 5-minute rest period was given between successive CMGs to allow the distended bladder to recover. Although the pigs were implanted bilaterally (for another study), only unilateral stimulation was used in this study.

\section{Statistical Analysis}

IBM SPSS Statistics ver. 19.0 (IBM Co., Armonk, NY, USA) was used for the statistical analysis. The significance level was set at $\mathrm{P}<0.05$. To compare the repeated CMG recordings, the BCs were normalized to the measurement of the first control CMG during NS. Repeated measurements of the same animal under the same CMG conditions were averaged. All summary data were expressed as mean \pm standard error. Statistical significance was detected by the Student $\mathrm{t}$-test or 1-way analysis of variance followed by the least significant difference test.

\section{RESULTS}

After 1 week, acute SNM experiments were performed. Irritation of the bladder with 5\% AA induced bladder overactivity and significantly reduced the $\mathrm{BC}$ to $34.4 \% \pm 4.7 \%$ of the NS control level $(354.4 \pm 35.9 \mathrm{~mL})(\mathrm{P}<0.05)$ (Figs. 4, 5). All pigs showed perianal and/or tail movements when the stimulator was turned on. During AA infusion, SNM at the $5-\mathrm{Hz}$ frequency did not significantly change the BC $(48.1 \% \pm 6.9 \%$ of the NS control level) compared with the AA level $(\mathrm{P}>0.05)$, but SNM at $15-, 30-$, and $50-\mathrm{Hz}$ frequencies significantly increased the $\mathrm{BC}$ to $54.5 \% \pm 7.1 \%, 55.2 \% \pm 6.5 \%$, and $57.2 \% \pm 6.1 \%$ of the NS control level compared with the AA level $(\mathrm{P}<0.05)$, respectively (Figs. 4, 5). No significant differences were present in the results obtained using those 3 frequencies (Figs. 4, 5). When examined at $30-$ and $50-\mathrm{Hz}$ frequencies, all pigs showed stiff tails.

\section{DISCUSSION}

It has been reported that, under physiological conditions, bladder distention by NS infusion primarily activates nonnociceptive bladder $\mathrm{A} \delta$-afferent fibers, which trigger supraspinal micturition. However, under pathological conditions, bladder irritation by AA infusion activates nociceptive $\mathrm{C}$-afferent fibers, which induce a spinal micturition reflex [15]. In this study, AA induced overactivity and reduced BC compared with the NS control levels (Figs. 4, 5). The sacral nerve was activated by electrical stimulation over a range of frequencies so that frequency response curves could be compared (Figs. 4, 5). We found that $\mathrm{SNM}$ at the $5-\mathrm{Hz}$ frequency did not significantly change the $\mathrm{BC}$, while SNM at the 15-, 30-, and 50-Hz frequencies significantly increased the $\mathrm{BC}$ during AA infusion. Moreover, no significant differences were observed in the results obtained using those 3 frequencies. This indicates that stimulation at $15 \mathrm{~Hz}$ seems to be sufficient to inhibit bladder activity. SNM is not frequency-dependent in the range of $15-50 \mathrm{~Hz}$. Currently, the 


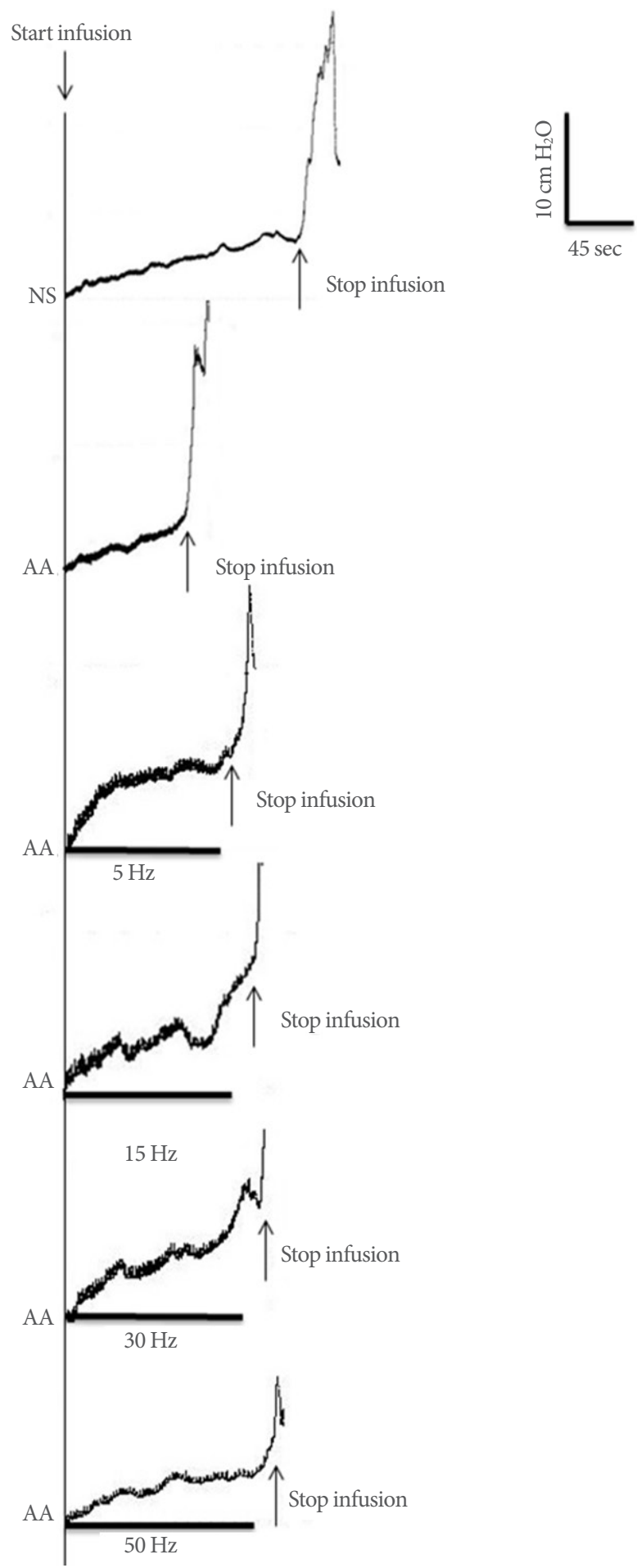

Fig. 4. Sacral nerve stimulation was given over a range of frequencies to suppress bladder overactivity induced by intravesical infusion of $5 \%$ acetic acid. The black bars under pressure trace indicate the stimulation duration. The short arrows indicate the start and stop of bladder infusions. Stimulation: pulse width $210 \mu \mathrm{sec}$, intensity $4 \mathrm{~V}$. Infusion rate: $30 \mathrm{~mL} / \mathrm{min}$.

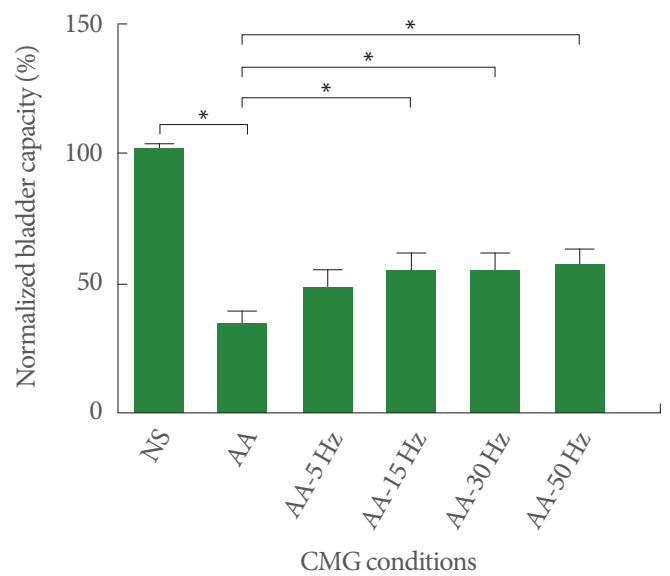

Fig. 5. Sacral nerve stimulation inhibited bladder overactivity during $5 \%$ acetic acid infusion at 15 to $50 \mathrm{~Hz}$. There were no significant differences among $15-, 30-$, and $50-\mathrm{Hz}$ frequencies $(\mathrm{P}>0.05)$. Stimulation: pulse width $210 \mu \mathrm{sec}$, intensity $4 \mathrm{~V}$. NS, normal saline; AA, acetic acid; CMG, cystometrogram. ${ }^{*}$ Statistically significant difference.

frequency used in the clinical practice of SNM is $10-20 \mathrm{~Hz} ; 14$ $\mathrm{Hz}$ is recommended [4-7]. It is assumed that the inhibitory effect of SNM on bladder activity is partially due to the activation of the large-diameter pudendal afferent nerves in the sacral dorsal roots [10]. However, Sawan et al. [16] claimed that complete bladder emptying could be achieved by stimulating the sacral spinal roots at frequencies of $300-350 \mathrm{~Hz}$ to fatigue the external urethral sphincter. Whether higher stimulation frequencies of SNM can excite the bladder remains to be investigated.

Tai et al. [8] showed that TNS at either low $(5 \mathrm{~Hz})$ or high (30 $\mathrm{Hz}$ ) frequencies inhibited the bladder overactivity induced by $\mathrm{AA}$ infusion in cats and significantly increased the BC compared with the AA control level. They stated that the $20-\mathrm{Hz}$ frequency currently used in the clinical applications of TNS seemed to be unnecessary because $5-\mathrm{Hz}$ stimulation could achieve the same therapeutic effects as $20-\mathrm{Hz}$ stimulation. Another study demonstrated that the activation of somatic afferent nerves in the foot could inhibit bladder overactivity in cats at either low $(5 \mathrm{~Hz})$ or high $(20 \mathrm{~Hz})$ frequencies [9]. Some recent studies have demonstrated PNS to be frequency-dependent $[10,12,17,18]$. PNS at low frequencies $(5-10 \mathrm{~Hz})$ inhibited bladder activity, while high frequencies $(20-30 \mathrm{~Hz})$ induced an excitatory reflex. They speculated that the frequency response characteristics of PNS might be related to the inability of synaptic transmission in the inhibitory pathway at high frequencies 
[12]. Although the mechanism of this pattern of frequency-dependence is still unknown, different stimulation frequencies must activate different spinal micturition reflex circuitry [19]. In addition, the release of different neurotransmitters at the first spinal synapse between the primary afferent axons and spinal interneurons might be triggered by different stimulation frequencies [17]. Observations of different outcomes for various nerve stimulation patterns indicate that the underlying mechanisms among them might be significantly different.

To the best of our knowledge, few studies have been conducted on SNM in large animal models using an implanted, clinically used stimulator, except a study using the InterStimII neurostimulator (Medtronic, Minneapolis, MN, USA) in sheep [20]. We used BC as a quantitative metric, mimicking clinical cystometry, which is performed in patients. Moreover, studies on the effects of different frequencies of SNM on bladder overactivity are rare. We provide an experimental basis for the further study of SNM in clinical practice. Although the maximum output frequency of the stimulator used in this study was 110 $\mathrm{Hz}$, we did not investigate that frequency. Some studies have reported the use of higher frequencies $(1-10 \mathrm{kHz}$ for PNS and $300-350 \mathrm{~Hz}$ for SNM) in animals $[10,16,19]$. However, these frequencies are designed for experimental research, and whether it is practical to use them for SNM in clinical practice remains to be examined. Whether acute and chronic SNM require different frequencies remains to be explored in future studies. It is also necessary to determine whether specific stimulation frequencies are safe for long-term use.

In summary, this study demonstrated that $\mathrm{SNM}$ at $15 \mathrm{~Hz}$ was an appropriate frequency, and that frequencies higher than 15 $\mathrm{Hz}$ did not lead to better surgical outcomes.

\section{REFERENCES}

1. D'Souza AO, Smith MJ, Miller LA, Doyle J, Ariely R. Persistence, adherence, and switch rates among extended-release and immediate-release overactive bladder medications in a regional managed care plan. J Manag Care Pharm 2008;14:291-301.

2. Aboseif S, Tamaddon K, Chalfin S, Freedman S, Kaptein J. Sacral neuromodulation as an effective treatment for refractory pelvic floor dysfunction. Urology 2002;60:52-6.

3. Fall M, Lindström S. Electrical stimulation. A physiologic approach to the treatment of urinary incontinence. Urol Clin North Am 1991;18:393-407.

4. Seif C, Eckermann J, Bross S, Portillo FJ, Jünemann KP, Braun PM.
Findings with bilateral sacral neurostimulation: sixty-two PNETests in patients with neurogenic and idiopathic bladder dysfunctions. Neuromodulation 2004;7:141-5.

5. Chartier-Kastler EJ, Ruud Bosch JL, Perrigot M, Chancellor MB, Richard F, Denys P. Long-term results of sacral nerve stimulation (S3) for the treatment of neurogenic refractory urge incontinence related to detrusor hyperreflexia. J Urol 2000;164:1476-80.

6. Groen J, Blok BF, Bosch JL. Sacral neuromodulation as treatment for refractory idiopathic urge urinary incontinence: 5 -year results of a longitudinal study in 60 women. J Urol 2011;186:954-9.

7. Chaabane W, Guillotreau J, Castel-Lacanal E, Abu-Anz S, De Boissezon X, Malavaud B, et al. Sacral neuromodulation for treating neurogenic bladder dysfunction: clinical and urodynamic study. Neurourol Urodyn 2011;30:547-50.

8. Tai C, Chen M, Shen B, Wang J, Roppolo JR, de Groat WC. Irritation induced bladder overactivity is suppressed by tibial nerve stimulation in cats. J Urol 2011;186:326-30.

9. Tai C, Shen B, Chen M, Wang J, Liu H, Roppolo JR, et al. Suppression of bladder overactivity by activation of somatic afferent nerves in the foot. BJU Int 2011;107:303-9.

10. Tai C, Smerin SE, de Groat WC, Roppolo JR. Pudendal-to-bladder reflex in chronic spinal-cord-injured cats. Exp Neurol 2006;197: 225-34.

11. Ju YH, Liao LM. Electrical stimulation of dog pudendal nerve regulates the excitatory pudendal-to-bladder reflex. Neural Regen Res 2016;11:676-81.

12. Tai C, Chen M, Shen B, Wang J, Liu H, Roppolo JR, et al. Plasticity of urinary bladder reflexes evoked by stimulation of pudendal afferent nerves after chronic spinal cord injury in cats. Exp Neurol 2011;228:109-17.

13. Andersen IS, Rijkhoff NJ, Vukovic A, Buntzen S, Djurhuus JC, Laurberg $S$. Anorectal motility responses to selective stimulation of the ventral sacral nerve roots in an experimental model. Br J Surg 2005;92:1513-9.

14. Schäfer W, Abrams P, Liao L, Mattiasson A, Pesce F, Spangberg A, et al. Good urodynamic practices: uroflowmetry, filling cystometry, and pressure-flow studies. Neurourol Urodyn 2002;21:261-74.

15. Fowler CJ, Griffiths D, de Groat WC. The neural control of micturition. Nat Rev Neurosci 2008;9:453-66.

16. Sawan M, Hassouna MM, Li JS, Duval F, Elhilali MM. Stimulator design and subsequent stimulation parameter optimization for controlling micturition and reducing urethral resistance. IEEE Trans Rehabil Eng 1996;4:39-46.

17. Wang J, Liu H, Shen B, Roppolo JR, de Groat WC, Tai C. Bladder inhibition or excitation by electrical perianal stimulation in a cat 
model of chronic spinal cord injury. BJU Int 2009;103:530-6.

18. Yang G, Wang J, Shen B, Roppolo JR, de Groat WC, Tai C. Pudendal nerve stimulation and block by a wireless-controlled implantable stimulator in cats. Neuromodulation 2014;17:490-6.

19. Tai C, Wang J, Wang X, Roppolo JR, de Groat WC. Voiding reflex in chronic spinal cord injured cats induced by stimulating and blocking pudendal nerves. Neurourol Urodyn 2007;26:879-86.

20. Brink TS, Zimmerman PL, Mattson MA, Su X, Nelson DE. A chronic, conscious large animal platform to quantify therapeutic effects of sacral neuromodulation on bladder Function. J Urol 2015;194:252-8. 